\title{
Analyse der strahleninduzierten Elektrolytveränderungen in Nierenschnitten
}

\author{
Von H. Breuer und H. K. Parchwitz \\ Aus der Chirurgischen Universitätsklinik und Poliklinik Bonn \\ (Z. Naturforschg. 15 b, 671—675 [1960] ; eingegangen am 19. März 1960)
}

\begin{abstract}
The effect of X-irradiation $(14000 \mathrm{r}-58000 \mathrm{r})$ on the movements of potassium and sodium in guinea pig kidney cortex slices after incubation under various conditions has been investigated. In addition, the oxygen uptake of the tissue slices has been measured.

The concentration gradients of potassium and sodium between slices and medium were reduced after X-irradiation. However, this reduction was only found when the slices were incubated at $37{ }^{\circ} \mathrm{C}$. At lower temperatures no effect of $\mathrm{X}$-irradiation was observed. The oxygen uptake of the kidney cortex slices remained unaffected by X-irradiation.

The experiments described in the present paper suggest that X-irradiation acts on the ion transport mechanism itself rather than on energy supplying processes.
\end{abstract}

Werden Nierencortex-Schnitte von Meerschweinchen in vitro einer Röntgenbestrahlung $\left(3 \cdot 10^{5} \mathrm{r}\right.$ bis $12 \cdot 10^{5} \mathrm{r}$ ) unterworfen und anschließend bei $37^{\circ}$ in K rebs-Ring e r-Phosphatlösung inkubiert, so beobachtet man gegenüber unbestrahlten Schnitten eine erhebliche Abnahme des Kaliumgehaltes und eine Zunahme des Natriumgehaltes; gleichzeitig wird die Atmung der Schnitte durch die genannten Dosen deutlich gesteigert ${ }^{1}$. Diese Ergebnisse führten zu der Arbeitshypothese, daß in Nierenschnitten die strahlenbedingte Beeinträchtigung des aktiven Transportes von Kalium und Natrium bei gleichzeitiger Steigerung der Atmung auf einer Veränderung der Zellpermeabilität bzw. der Permeationsvorgänge beruht.

In der vorliegenden Arbeit wurde nunmehr die Wirkung niedrigerer Strahlendosen $(14000 \mathrm{r}$ bis 58000 r) auf den Kalium- und Natriumgehalt von Nierencortex-Schnitten geprüft. Von Interesse erschien in diesem Zusammenhang eine genauere Untersuchung der Zeit- und Temperaturabhängigkeit der strahlenbedingten Elektrolyt-Veränderungen.

\section{Methodik}

Tie re und Ge we be. $400-500$ g schwere männliche Meerschweinchen wurden durch Dekapitation getötet und nach dem Ausbluten die Nieren entnommen. Die Herstellung der $0,3-0,4 \mathrm{~mm}$ dünnen Gewebeschnitte erfolgte nach der Methode von Deutsch ${ }^{2}$. Es wurden nur Schnitte der Nierencortex verwendet.

1 H. Breuer u. H. K. Parchwitz, Strahlentherapie 110, 451 [1959].

2 W. Deutsch, J. Physiology 87, 56 P [1936].
B estrahlung st e chnik. Nach dem Wägen wurden die Schnitte in $\mathrm{O}_{2}$-gesättigter $\mathrm{Krebs}-\mathrm{R}$ in $\mathrm{g}$ e $\mathrm{r}$ Phosphatlösung, $p_{\mathrm{H}} 7,4$, die $10 \mathrm{mMole} \alpha$-Ketoglutarat enthielt, für $5 \mathrm{~min}$ bei $+1^{\circ}$ gehalten („Vorinkubation"). Während dieser Zeit erfolgte die Bestrahlung, wobei Dosen von $14000 \mathrm{r}, 28000 \mathrm{r}$ und $56000 \mathrm{r}$ unter den früher angegebenen Bedingungen (s. l.c. ${ }^{1}$ ) appliziert wurden.

Inkubation und Bestimmung von $\mathrm{Na}$ trium und Kalium. Nach der „Vorinkubation“ wurden die bestrahlten und unbestrahlten Schnitte in eine frische Salzlösung überführt und bei $37^{\circ}$ inkubiert. Weitere Einzelheiten der Inkubation und flammenphotometrischen Bestimmung der Elektrolyte s. l. c. ${ }^{1}$.

Die Bestimmung der Atmung erfolgte nach der Methode von W ARBurg $^{3}$.

Die Größe des Inulinraumes wurde nach den Angaben von RoBinson ${ }^{4}$ bestimmt (vgl. l. c. ${ }^{5}$ ).

\section{Ergebnisse}

Vor Inkubation betrug der Kaliumgehalt der Nierencortex-Schnitte erwachsener Meerschweinchen 79,0 $\mu$-Äquiv./g Frischgewicht und der Natriumgehalt 58,0 $\mu$-Äquiv./g. Während der 45-min. Inkubation bei $37^{\circ}$ in $\alpha$-ketoglutarat-haltiger $\mathrm{K} \mathrm{rebs}$ Ringer - Phosphatlösung blieb der Kaliumgehalt der Schnitte praktisch unverändert $(76,0-79,0$ $\mu$-Äquiv./g), während ihr Natriumgehalt auf 90,0 bis 92,0 $\mu$-Äquiv./g anstieg (Tab. I). Daraus geht hervor, daß unter den oben genannten Bedingungen zwischen den Schnitten und der umgebenden Salzlösung ein normaler Konzentrationsgradient für

3 O. WARBURG, Biochem. Z. 142, 317 [1923].

4 J. R. Robinson, Proc. Roy. Soc. [London] Abt. B 137, 378 [1950].

5 H. Breuer u. R. Whittam, J. Physiology 135, 213 [1957]. 


\begin{tabular}{|c|c|c|c|c|c|}
\hline \multirow[b]{2}{*}{ Dosis in $r$} & \multirow[b]{2}{*}{$\begin{array}{c}\text { Inkubations- } \\
\text { temp. }\end{array}$} & \multicolumn{2}{|c|}{$\begin{array}{l}\text { Kaliumgehalt } \\
\text { [ } \mu \text {-Äquiv./g] }\end{array}$} & \multicolumn{2}{|c|}{$\begin{array}{c}\text { Natriumgehalt } \\
{[\mu \text {-Äquiv./g] }}\end{array}$} \\
\hline & & $\begin{array}{c}\text { unbestrahlte } \\
\text { Schnitte }\end{array}$ & $\begin{array}{l}\text { bestrahlte } \\
\text { Schnitte }\end{array}$ & $\begin{array}{l}\text { unbestrahlte } \\
\text { Schnitte }\end{array}$ & $\begin{array}{c}\text { bestrahlte } \\
\text { Schnitte }\end{array}$ \\
\hline $\begin{array}{l}14000 \\
28000 \\
58000\end{array}$ & $\begin{array}{l}37^{\circ} \\
37^{\circ} \\
37^{\circ}\end{array}$ & $\begin{array}{l}76,0(5) \\
78,0(6) \\
79,5(5)\end{array}$ & $\begin{array}{l}69,0(6) \\
63,0(6) \\
61,0(6)\end{array}$ & $\begin{array}{l}91,5(5) \\
90,0(6) \\
92,0(6)\end{array}$ & $\begin{array}{r}98,0(6) \\
101,0(6) \\
104,0(6)\end{array}$ \\
\hline $\begin{array}{l}14000 \\
28000 \\
58000\end{array}$ & $\begin{array}{l}+1^{\circ} \\
+1^{\circ} \\
+1^{\circ}\end{array}$ & $\begin{array}{l}31,0(5) \\
33,0(6) \\
37,5(6)\end{array}$ & $\begin{array}{l}34,0(6) \\
31,0(6) \\
33,0(6)\end{array}$ & $\begin{array}{l}113,5(6) \\
120,0(6) \\
114,0(6)\end{array}$ & $\begin{array}{l}118,1(6) \\
112,5(6) \\
122,5(6)\end{array}$ \\
\hline \multicolumn{2}{|c|}{ Ausgangsgewebe } & \multicolumn{2}{|c|}{$79,0(8)$} & \multicolumn{2}{|c|}{$60,5(8)$} \\
\hline
\end{tabular}

Tab. 1. Wirkung der Röntgenbestrahlung auf den Kalium- und Natriumgehalt von Nierencortex-Schnitten erwachsener Meerschweinchen nach Inkubation in $\mathrm{K} \mathrm{r} \mathrm{e} \mathrm{b} \mathrm{s} \mathrm{-} \mathrm{R} \mathrm{i} \mathrm{n} \mathrm{g} \mathrm{e} \mathrm{r} \mathrm{-} \mathrm{Phosphatlösung} \mathrm{bei} 37^{\circ}$ und bei $+1^{\circ} .50-100$ mg Gewebe wurden in $5,0 \mathrm{ml} \mathrm{O} \mathrm{O}_{2}$-gesättigter $\mathrm{K} \mathrm{r} \mathrm{e} \mathrm{b} \mathrm{s-R} \mathrm{in} \mathrm{g} \mathrm{e} \mathrm{r-Phosphatlösung} \mathrm{von} p_{\mathrm{H}} 7,4$, die $10 \mathrm{mMole}$ - -Ketoglutarat als Substrat enthielt, für $45 \mathrm{~min}$ bei $37^{\circ}$ bzw. $+1^{\circ}$ inkubiert. Die Bestrahlung der Schnitte erfolgte bei $+1^{\circ}$. Die Zahlen in der Tab. 1 geben die Mittelwerte sowie die Anzahl der Versuche an.

Kalium aufrecht erhalten wurde; dagegen war bedingt durch die Zunahme des Natriumgehaltes eine Abnahme des Natriumgradienten nicht zu verhindern. Nach 45-min. Inkubation bei $+1^{\circ}$ war der Kaliumgehalt der Schnitte auf etwa $30 \mu$-Äquiv./g abgesunken; andererseits war ihr Natriumgehalt auf das Doppelte des Ausgangswertes angestiegen.

Wenn die Schnitte einer Röntgenbestrahlung $(14000 \mathrm{r}-58000 \mathrm{r}$ ) unterworfen wurden, verloren sie während der sich anschließenden Inkubation bei $37^{\circ}$ einen Teil ihres Kaliumgehaltes. Wie aus Tab. 1 hervorgeht, nahm der Kaliumgehalt mit zunehmender Bestrahlungsdosis ab. Nach einer Dosis von

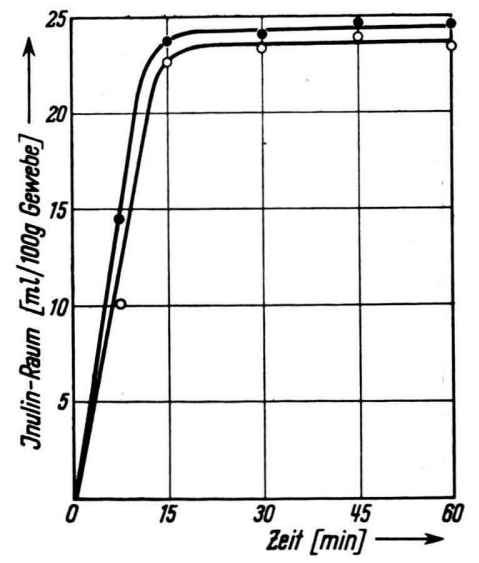

Abb. 1. Inulinraum unbestrahlter und bestrahlter Nierencortex-Schnitte männlicher Meerschweinchen. Die Schnitte wurden in $\mathrm{O}_{2}$-gesättigter $\mathrm{Kr}$ e b s- $\mathrm{R}$ in ge r-Phosphatlösung von $p_{\mathrm{H}} 7,4$, die $10 \mathrm{mMole} \alpha$-Ketoglutarat als Substrat enthielt, bei $37^{\circ}$ inkubiert; die Inulinkonzentration in der Salzlösung betrug 1 Prozent. Die Schnitte wurden zu verschiedenen Zeiten nach Versuchsbeginn entnommen. Jeder Kurvenpunkt ist ein Mittelwert aus 3 Einzelbestimmungen. ०: unbestrahlte Schnitte, $\bullet$ : mit $58000 \mathrm{r}$ bestrahlte Schnitte.
$58000 \mathrm{r}$ betrug der Kaliumverlust etwa $18 \mu$ Äquiv./g. Dieser Verlust wurde durch eine Aufnahme von Natrium teilweise kompensiert. So stieg der Natriumgehalt nach Bestrahlung der Schnitte mit $58000 \mathrm{r}$ gegenüber den Kontrollen um etwa $12 \mu$-Äquiv./g an. Wurden die bestrahlten Schnitte für 45 min bei $+1^{\circ}$ inkubiert, so blieb ihr Elektrolytgehalt gegenüber den unbestrahlten Schnitten praktisch unverändert (Tab. 1).

Um von den Elektrolyt-Konzentrationen im Gesamtschnitt auf die Konzentrationen in den Cortexzellen schließen zu können, ist es notwendig, die Ausdehnung des extracellulären Raumes zu kennen; man kann dann unter der Annahme, daß die ionale Zusammensetzung des extracellulären Raumes derjenigen des suspendierenden Mediums gleich ist, den Elektrolytgehalt der Cortexzellen - also des intracellulären Raumes - berechnen. Aus diesem Grunde wurde der Inulinraum, der als identisch mit der Größe des extracellulären Raumes gilt, in unbestrahlten und bestrahlten Nierencortex-Schnitten bestimmt. In Abb. 1 ist der zeitliche Verlauf der Inulinaufnahme durch Nierencortex-Schnitte dargestellt. Jeder Punkt der Kurven ist ein Mittelwert aus 3 Bestimmungen. Bereits nach 15-min. Inkubation waren die Schnitte mit Inulin gesättigt. Die Mittelwerte des Inulinraumes betrugen in der Zeitspanne von 30 bis $60 \mathrm{~min}$ für unbestrahlte Schnitte $23,6 \mathrm{ml} / 100 \mathrm{~g}$ Frischgewicht und für Schnitte, die mit $58000 \mathrm{r}$ bestrahlt worden waren, $24,2 \mathrm{ml} / 100 \mathrm{~g}$ Frischgewicht. Die in-vitro-Bestrahlung der NierencortexSchnitte hatte also keinen erkennbaren Einfluß auf die Größe des extracellulären Raumes.

Der zeitliche Verlauf der. Elektrolytänderungen 


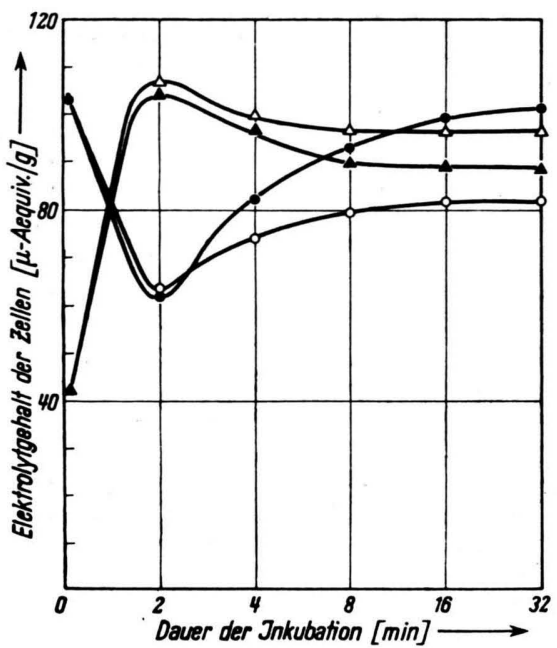

Abb. 2. Zeitlicher Verlauf der Elektrolyt-Veränderungen in unbestrahlten und mit $58000 \mathrm{r}$ bestrahlten NierencortexSchnitten männlicher Meerschweinchen während einer Inkubation bei $37^{\circ} .50-100 \mathrm{mg}$ Schnitte wurden in $\mathrm{O}_{2}$-gesättigter $\mathrm{Krebs}-\mathrm{R}$ ing e r - Phosphatlösung von $p_{\mathrm{H}} 7,4$, die 10 mMole $\alpha$-Ketoglutarat als Substrat enthielt, für 2, 4, 8, 16 bzw. 32 min inkubiert. Jeder Kurvenpunkt ist ein Mittelwert aus 3 Einzelbestimmungen. - Kaliumgehalt unbestrahlter Schnitte; o: Kaliumgehalt bestrahlter Schnitte; $\boldsymbol{\Delta}$ : Natriumgehalt unbestrahlter Schnitte; $\triangle:$ Natriumgehalt bestrahlter Schnitte.

in unbestrahlten und bestrahlten Nierencortex-Zellen ist in Abb. 2 dargestellt. Unter Berücksichtigung der Größe des extracellulären Raumes sind alle Werte in $\mu$-Äquiv./g Zellen angegeben. Wie bereits Whitтaм und Davies ${ }^{6}$ beobachtet hatten, verloren die Cortexzellen während der ersten Min. der Inkubation einen beträchtlichen Teil ihres Kaliumgehaltes und nahmen gleichzeitig Natrium aus der Salzlösung auf. Diese Änderungen, die offenbar durch die Verletzung des Gewebes beim Herstellen der Schnitte bedingt waren und durch den hohen Natrium- und niedrigen Kaliumgehalt des Inkubationsmediums begünstigt wurden, führten zu einer Abnahme der KonzentrationsGradienten. Nach diesen anfänglichen Veränderungen begannen die Zellen, Kalium wieder zu akkumulieren und Natrium abzuschieben. Im Zeitraum zwischen 10 und $60 \mathrm{~min}$ trat dann hinsichtlich des Kalium- und Natriumgehaltes ein Gleichgewichtszustand ein. Die zeitlichen Änderungen des Elektrolytgehaltes in den bestrahlten Schnitten waren ähnlich denjenigen in unbestrahlten Geweben. Auch hier kam es zunächst zu einem Kaliumverlust und einer Natriumaufnahme, die jedoch - wenn auch in ge-

\footnotetext{
6 R. Whittam u. R. E. Davies, Biochem. J. 55, 880 [1953].
}

ringerem Umfange - nach 10 min wieder rückläufig waren. Im Gegensatz zu den Kontrollen blieb in den bestrahlten Cortexzellen während der folgenden $40-50$ min der Kaliumgehalt erniedrigt und der Natriumgehalt erhöht.

Die Aufrechterhaltung normaler KonzentrationsGradienten zwischen dem intra- und extracellulären Raum ist von einer ausreichenden Energiezufuhr abhängig. Es erschien deshalb interessant, das Verhalten von Kalium und Natrium in bestrahlten Cortexschnitten nach Inkubation bei verschiedenen Temperaturen zu prüfen. Die Ergebnisse sind in Abb. 3 dargestellt. Auffallend ist, daß die höchsten Kaliumwerte nach Inkubation nicht etwa bei $37^{\circ}$, sondern bei $27^{\circ}$ beobachtet wurden. Das gleiche gilt - mit umgekehrtem Vorzeichen - auch für Natrium. Sowohl in unbestrahlten Schnitten als auch in bestrahlten Schnitten war der Kaliumgehalt nach Inkubation bei $+1^{\circ}$ am geringsten. Erfolgte die Inkuba-

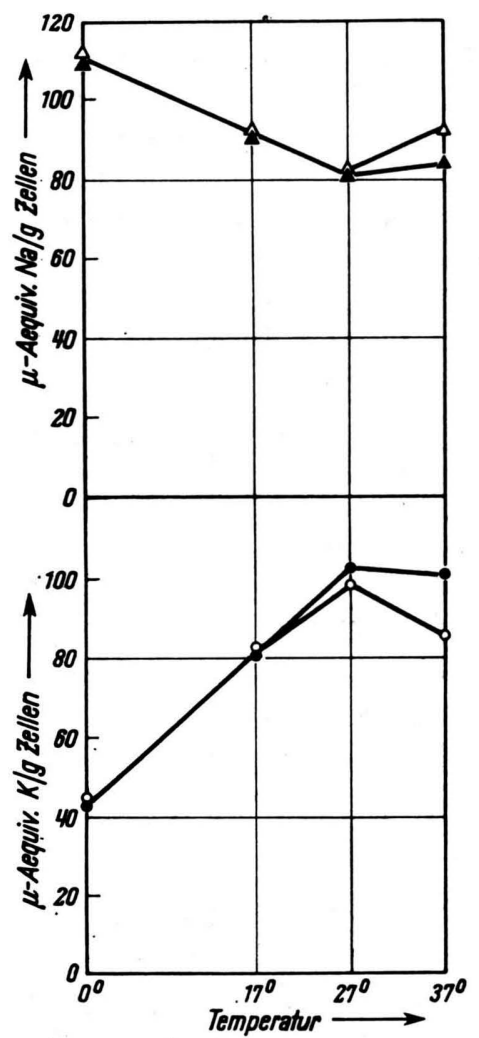

Abb. 3. Abhängigkeit des Kalium- und Natriumgehaltes unbestrahlter und mit $58000 \mathrm{r}$ bestrahlter Nierencortexzellen von der Temperatur. Jeder Kurvenpunkt ist ein Mittelwert aus mindestens 3 Bestimmungen. $\bullet$ : Kaliumgehalt unbestrahlter Cortexzellen; O: Kaliumgehalt bestrahlter Cortexzellen; $\Delta$ : Natriumgehalt unbestrahlter Cortexzellen; $\triangle$ : Natriumgehalt bestrahlter Cortexzellen. 
tion bei $17^{\circ}$, so wurde zwischen unbestrahlten und bestrahlten Cortexzellen kein Unterschied im Elektrolytgehalt festgestellt. Nach Inkubation bei $27^{\circ}$ traten nur geringfügige Unterschiede zwischen bestrahlten und unbestrahlten Schnitten auf. Der strahlenbedingte Verlust von Kalium und die erhöhte Aufnahme von Natrium kamen erst voll zur Wirkung, wenn das Gewebe bei $37^{\circ}$ inkubiert wurde.

Da die Atmung als Energiequelle für den aktiven Transport gilt, wurde die Sauerstoffaufnahme unbestrahlter und bestrahlter Schnitte gemessen. Die Atmung normaler Nierencortex-Schnitte zeigte die bekannte Temperaturabhängigkeit enzymatischer Prozesse: so betrug der $Q_{\mathrm{O}_{2}}\left(-\mu \mathrm{lO}_{2} / \mathrm{mg} \cdot \mathrm{Std}\right.$. $)$ in a-ketoglutarat-haltiger $\mathrm{K} \mathrm{r} \mathrm{e} \mathrm{b} \mathrm{s-R} \mathrm{in} \mathrm{g} \mathrm{e} \mathrm{r-Phosphat-}$ lösung $-4,9$ bei $17^{\circ},-9,2$ bei $27^{\circ}$ und $-18,0$ bei $37^{\circ}$. Im Bereich von $0^{\circ}$ bis $27^{\circ}$ war die zunehmende $\mathrm{O}_{2}$-Aufnahme von einer entsprechenden Kaliumzunahme begleitet. Zwischen $27^{\circ}$ und $37^{\circ}$ stieg zwar die Atmung weiterhin an, doch nahm der Kaliumgehalt der Schnitte - wie bereits erwähnt - in diesem Temperaturbereich wieder etwas ab. Eine Bestrahlung mit $14000 \mathrm{r}, 28000 \mathrm{r}$ und $56000 \mathrm{r}$ hatte keine erkennbare Wirkung auf die Sauerstoffaufnahme der Schnitte bei $17^{\circ}, 27^{\circ}$ und $37^{\circ}$.

\section{Besprechung der Ergebnisse}

Die Versuche zeigen, daß eine Bestrahlung mit $14000 \mathrm{r}-58000 \mathrm{r}$ die Aufrechterhaltung der Konzentrations-Gradienten für Kalium und Natrium in Nierencortex-Schnitten bei Inkubation in physiologischen Lösungen beeinträchtigt. Im Gegensatz dazu bleibt die $\mathrm{O}_{2}$-Aufnahme der Gewebeschnitte durch die Bestrahlung unbeeinflußt. Dieser Befund deutet bereits darauf hin, daß die Elektrolyt-Verschiebungen in dem genannten Dosisbereich offenbar nicht durch eine Hemmung der am aktiven Transport beteiligten energieliefernden Vorgänge zustande kommt. Ein weiterer Hinweis für diese Annahme ist die Beobachtung, daß die strahlenbedingte Beeinflussung der Elektrolyt-Verschiebungen erst nach Inkubation der Schnitte bei $37^{\circ}$ voll zur Wirkung kommt. Bei einer direkten Schädigung der mit dem aktiven Transport gekoppelten energieliefernden Prozesse würde man dagegen bereits bei $17^{\circ}$ und $27^{\circ}$ eine Beeinträchtigung des Elektrolytstoffwechsels erwarten.

Der fehlende Unterschied im Kalium- und Natriumgehalt bestrahlter und unbestrahlter Schnitte während der Inkubation bei $+1^{\circ}$ beruht darauf, daß bei dieser Temperatur die Verteilung der Elektrolyte zwischen dem Gewebe und der Salzlösung durch die D o n n a n - Gleichgewichte beherrscht wird und dabei biologische Prozesse ausgeschaltet sind.

Als besondere Eigenart der Nierenschnitte sei erwähnt, daß sie während der Inkubation bei $27^{\circ}$ mehr Kalium akkumulieren als bei $37^{\circ}$. Eine ähnliche Beobachtung machte Mudge ${ }^{7}$ bei Nierenschnitten von Kaninchen. Mudge äußerte die Vermutung, da $\beta$ der Kaliumgradient zwischen dem Gewebe und dem umgebenden Milieu von zwei Faktoren abhängig ist, nämlich von der Aktivität des Stoffwechsels und von der Integrität der Zelle. Höhere Temperaturen - etwa $37^{\circ}$ - sollen entweder den Abbau wichtiger cellulärer Komponenten beschleunigen oder eine relative Anoxie herbeiführen, so daß die Ausbildung eines maximalen Konzentrations-Gradienten verhindert wird. Die Tatsache, daß die strahlenbedingten Elektrolyt-Verschiebungen erst bei $37^{\circ}$ und nicht schon bei niedrigeren Temperaturen manifest werden, spricht gegen eine Alteration physikalischer Reaktionen, da diese in ihrem Ablauf temperaturunabhängig sind. Viel eher kann zur Erklärung des Temperatureffektes die Möglichkeit einer Beeinflussung biochemischer Prozesse herangezogen werden. Da eine Schädigung der für den aktiven Ionentransport notwendigen energieliefernden Vorgänge im vorliegenden Fall weitgehend ausgeschlossen werden konnte, liegt es nahe, eine Beeinträchtigung enzymatisch regulierter Permeationsvorgänge anzunehmen.

Die Untersuchung des zeitlichen Verlaufs der Elektrolyt-Verschiebungen ergab, daß der Kaliumabfall und die Natriumzunahme während der ersten min der Inkubation in den bestrahlten und unbestrahlten Schnitten praktisch gleich groß war. In der sich anschließenden Phase zeigten auch die bestrahlten Schnitte eine gewisse Tendenz, Kalium zu akkumulieren und Natrium abzuschieben, doch waren sie darin weniger erfolgreich als die unbestrahlten Schnitte. Diese Ergebnisse deuten darauf hin, daß der Transportmechanismus in den bestrahlten Schnitten nicht vollständig beschädigt oder gehemmt war.

Aus den vorliegenden Ergebnissen kann mit großer Wahrscheinlichkeit auf eine Beeinträchtigung des für den aktiven Kationentransport verantwortlichen Transportmechanismus durch die Röntgenbestrah-

7 G. H. Mudge, Amer. J. Physiology 165, 113 [1951]. 
lung geschlossen werden. Die Frage, ob die Hemmung des aktiven Transportes durch eine Schädigung von Trägermolekülen oder durch Veränderungen der Zellpermeabilität herbeigeführt wird, läßt sich noch nicht schlüssig beantworten; möglicherweise sind beide Faktoren eng miteinander gekoppelt. Eine strahlenbedingte Hemmung energieliefern- der Vorgänge dürfte für die hier beobachteten Elektrolyt-Verschiebungen nur eine untergeordnete Rolle spielen.

Die Untersuchungen wurden durch eine Forschungsbeihilfe des Bundesministeriums für A tomkernenergie und Wasserwirtschaft ermöglicht.

\section{NOTIZEN}

\section{Photochemische Darstellung von Cyclopentadienyl- und Methylcyclopenta- dienylmangandicarbonylpyridin}

\section{Von W. Strohmeier und KL. Gerlach}

Institut für Physikalische Chemie der Universität Würzburg (Z. Naturforschg. 15 b, 675-676 [1960] ; eingegangen am 4. August 1960)

Durch UV-Bestrahlung einer Lösung von Methylcyclopentadienyl- bzw. Cyclopentadienylmangantricarbonyl in Pyridin erhält man unter Abspal. tung von CO Methylcyclopentadienyl- bzw. Cyclopentadienylmangandicarbonylpyridin. Die Verbindungen können aus der Reaktionslösung in Form gelbbrauner, sublimierbarer und an der Luft beständiger Kristalle erhalten werden.

In Fortführung unserer Versuche zur photochemischen Darstellung von Derivaten der Metallcarbonyle ${ }^{1,2}$ versuchten wir, ob auch in Cyclopentadienylmetallcarbonylen unter dem Einfluß einer UV-Bestrahlung eine oder mehrere CO-Gruppen durch einen anderen Donator ersetzt werden können. Zu diesem Zwecke wurde eine Lösung von Cyclopentadienylmangantricarbonyl $\mathrm{C}_{5} \mathrm{H}_{5} \mathrm{Mn}(\mathrm{CO})_{3}$ in Pyridin $(\mathrm{Py})$ in der schon beschriebenen Versuchsanordnung ${ }^{2}$ solange mit UV-Licht bestrahlt, bis pro mMol eingesetzter Substanz ungefähr ein mMol CO abgespalten war. Dabei bildete sich nach der Reaktionsgleichung:

$$
\mathrm{C}_{5} \mathrm{H}_{5} \mathrm{Mn}(\mathrm{CO})_{3}+\mathrm{Py} \stackrel{h \nu}{\stackrel{n}{*}} \mathrm{C}_{5} \mathrm{H}_{5} \mathrm{Mn}(\mathrm{CO})_{2} \mathrm{Py}+\mathrm{CO}
$$

Cyclopentadienylmangandicarbonylpyridin, welches als sublimierbare, kristalline Substanz isoliert werden konnte. Die CO-Abspaltung ging am Anfang flott vonstatten, wurde dann aber immer langsamer und kam praktisch zum Stillstand, nachdem pro mMol eingesetzter Substanz ein mMol CO abgespalten war. Unter den unten angegebenen Versuchsbedingungen gelang es nicht noch eine weitere CO-Gruppe abzuspalten, um so zu einem Cyclopentadienylmangancarbonyldipyridin zu kommen.

Ganz analog verliefen die entsprechenden Versuche mit Methylcyclopentadienylmangantricarbonyl, welche Methylcyclopentadienylmangandicarbonylpyridin $\mathrm{C}_{6} \mathrm{H}_{7} \mathrm{Mn}(\mathrm{CO})_{2} \mathrm{Py}$ ergaben.

1 W. Strohmeier u. Kl. Gerlach, Z. Naturforschg. 15 b, 622, 413 [1960]; Chem. Ber. 93, 2087 [1960].
Während die Lösungen von $\mathrm{C}_{5} \mathrm{H}_{5} \mathrm{Mn}(\mathrm{CO})_{3}$ und $\mathrm{C}_{6} \mathrm{H}_{7} \mathrm{Mn}(\mathrm{CO})_{3}$ im Dunkeln gegen Luftsauerstoff beständig sind und nur photochemisch durch Sauerstoff oxydiert werden, zersetzen sich die Lösungen von $\mathrm{C}_{5} \mathrm{H}_{5} \mathrm{Mn}(\mathrm{CO}){ }_{2} \mathrm{Py}$ und $\mathrm{C}_{6} \mathrm{H}_{7} \mathrm{Mn}(\mathrm{CO}){ }_{2} \mathrm{Py}$ unter dem Einfluß des Luftsauerstoffs bereits im Dunkeln. $\mathrm{C}_{5} \mathrm{H}_{5} \mathrm{Mn}(\mathrm{CO}){ }_{2} \mathrm{Py}$ bildet gelbbraune nadelförmige Kristalle, welche an der Luft recht beständig sind. Die Substanz sublimiert im Hochvakuum bei $80-90^{\circ}$ und schmilzt bei $114^{\circ}$ (K of le r-Bank). Die Schmelze zersetzt sich an der Luft. Die Verbindung ist leicht löslich in Benzol, Alkohol und Aceton, schwer löslich in Petroläther und unlöslich in Wasser. Die Lösungen zersetzen sich rasch an der Luft.

$\mathrm{C}_{6} \mathrm{H}_{7} \mathrm{Mn}(\mathrm{CO}){ }_{2} \mathrm{Py}$ bildet ebenfalls gelbbraune nadelförmige Kristalle, welche bei $75^{\circ}$ schmelzen (K of le r Bank) und im Hochvakuum zwischen 80 und $9.0^{\circ}$ sublimieren. Beständigkeit, Löslichkeiten und $\mathbf{O x y}$ dationsempfindlichkeit entsprechen der Verbindung $\mathrm{C}_{5} \mathrm{H}_{5} \mathrm{Mn}(\mathrm{CO}){ }_{2} \mathrm{Py}$.

D a rstellung vo n $\mathrm{C}_{5} \mathrm{H}_{5} \mathrm{Mn}(\mathrm{CO}){ }_{2} \mathrm{Py}: 276,9 \mathrm{mg}$ $\mathrm{C}_{5} \mathrm{H}_{5} \mathrm{Mn}(\mathrm{CO})_{3}(1,35 \mathrm{mMole})$, gelöst in $25 \mathrm{ml}$ Pyridin, wurden bis zur Abspaltung von 1,16 mMolen CO bestrahlt. Nach dem Abziehen des Lösungsmittels bei Raumtemperatur im Hochvakuum verblieb ein gelbbrauner kristalliner Rückstand, welcher unter $\mathrm{N}_{2}$-Schutz in $2 \mathrm{ml}$ Methanol aufgenommen und in ein Sublimationsgefäß mit Kühlfinger überführt wurde. Nach dem Abziehen des Methanols im Ölpumpenvakuum bei Raumtemperatur sublimierte anschließend bei $50^{\circ}$ Badtemperatur im Hochvakuum noch etwas nicht umgesetzte Verbindung an den mit Leitungswasser gekühlten Kühlfinger. Die Sublimation wurde unterbrochen, der Kühlfinger gereinigt und die Hauptmenge des Produktes bei $80-90^{\circ}$ Badtemperatur im Hochvakuum auf den Kühlfinger sublimiert. Das so sublimierte Produkt wurde in der gleichen Weise nochmals resublimiert. Ausbeute an resublimiertem Produkt: $157 \mathrm{mg}=53 \%$ bezogen auf abgespaltenes $\mathrm{CO}$.

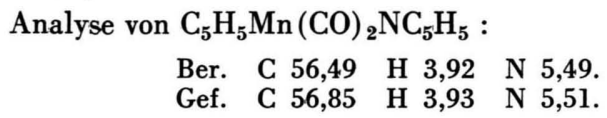

2 W.Strohmeier, KL. Gerlach u. G. Matthias, Z. Naturforschg. $15 \mathrm{~b}, 621$ [1960]. 